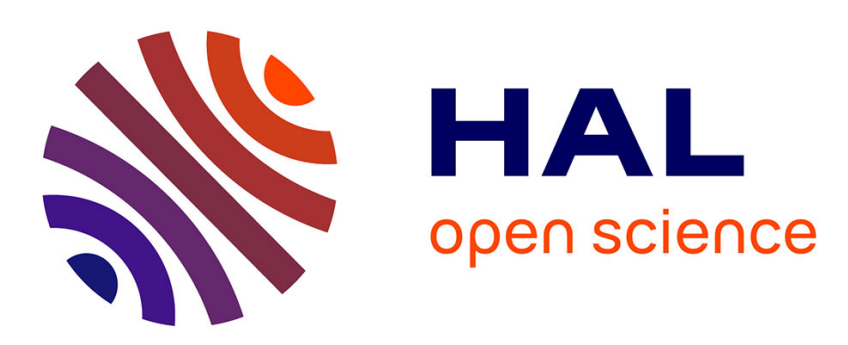

\title{
A mechanism of dynamical interactions for two-person social dilemmas
}

Krzysztof Mogielski, Tadeusz P!atkowski

\section{To cite this version:}

Krzysztof Mogielski, Tadeusz P!atkowski. A mechanism of dynamical interactions for two-person social dilemmas. Journal of Theoretical Biology, 2009, 260 (1), pp.145. 10.1016/j.jtbi.2009.06.007 . hal-00554626

\section{HAL Id: hal-00554626 \\ https://hal.science/hal-00554626}

Submitted on 11 Jan 2011

HAL is a multi-disciplinary open access archive for the deposit and dissemination of scientific research documents, whether they are published or not. The documents may come from teaching and research institutions in France or abroad, or from public or private research centers.
L'archive ouverte pluridisciplinaire HAL, est destinée au dépôt et à la diffusion de documents scientifiques de niveau recherche, publiés ou non, émanant des établissements d'enseignement et de recherche français ou étrangers, des laboratoires publics ou privés. 


\section{Author's Accepted Manuscript}

A mechanism of dynamical interactions for twoperson social dilemmas

Krzysztof Mogielski, Tadeusz Płatkowski

PII: $\quad$ S0022-5193(09)00259-8

DOI: $\quad$ doi:10.1016/j.jtbi.2009.06.007

Reference: $\quad$ YJTBI 5589

To appear in: $\quad$ Journal of Theoretical Biology

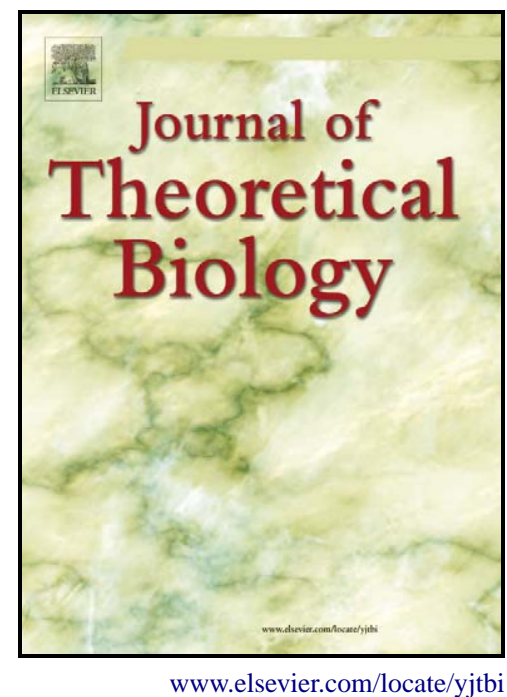

Received date: $\quad 8$ February 2009

Revised date: $\quad 31$ May 2009

Accepted date: $\quad 1$ June 2009

Cite this article as: Krzysztof Mogielski and Tadeusz P!atkowski, A mechanism of dynamical interactions for two-person social dilemmas, Journal of Theoretical Biology (2009), doi:10.1016/j.jtbi.2009.06.007

This is a PDF file of an unedited manuscript that has been accepted for publication. As a service to our customers we are providing this early version of the manuscript. The manuscript will undergo copyediting, typesetting, and review of the resulting galley proof before it is published in its final citable form. Please note that during the production process errors may be discovered which could affect the content, and all legal disclaimers that apply to the journal pertain. 


\title{
A Mechanism of Dynamical Interactions for Two-Person Social Dilemmas
}

\author{
Krzysztof Mogielski and Tadeusz Płatkowski* \\ Department of Mathematics, Informatics and Mechanics, \\ University of Warsaw, Banacha 2, 02-097 Warsaw, Poland
}

\begin{abstract}
We propose a new mechanism of interactions between game - theoretical agents in which the weights of the connections between interacting individuals are dynamical, payoff - dependent variables. Their evolution depends on the difference between the payoff of the agents from a given type of encounter and their average payoff. The mechanism is studied in the frame of two models: agents distributed on a random graph, and a mean field model. Symmetric and asymmetric connections between the agents are introduced. Long time behavior of both systems is discussed for the Prisoner's Dilemma and the Snow Drift games.
\end{abstract}

PACS numbers: 89.75.-k, 89.20.-a

Keywords: social dilemmas, coevolution on networks, mean field model

\section{INTRODUCTION}

In mathematical modeling of human societies the evolution of behaviors (actions) of the interacting individuals and of the structure of the mutual interactions should be taken into account. In real world the acceptance and refusal of entering in interactions with preferential partners is a common phenomenon. Real world networks change in time. People may tend to regulate the interpersonal interactions, connections with the others, on the basis of comparison between the results of the interactions and some averaged, local or global patterns. The players can change their actions and optimize the strength of relations with the others. In particular the players can change the structure of the links with the other players, breaking links and creating the new ones.

The models of populations of agents with interactions described by social dilemma games on spatial static regular lattices were introduced by Nowak and May (1992), and then studied by many authors, and extended to general spatial networks and structured populations. We refer to Szabo and Fath (2007), and Gross and Blasius (2008) for recent reviews on evolutionary games on graphs, and on adaptive coevolutionary networks.

*Electronic address: tplatk@mimuw.edu.pl 
The coevolution of network topology and strategy dynamics has been considered by many authors. Various concepts of the coevolving network structure and strategy distribution have been introduced, e.g. via assortative selection of interaction partners (cf. e.g. Ebel and Bornholdt, 2002; Eguiluz et al. 2005; Zimmermann and Eguiluz, 2005; Poncela et al., 2007; Ashlock et al., 1996, and references cited therein), volunteering participation (cf. e.g. Hauert and Szabo, 2003, and references cited therein), via random or intentional rewiring procedures (e.g. addition and/or removal of nodes, cf. e.g. Zimmermann et al., 2004), via introduction of different behaviors towards the adverse ties (Van Segbroeck et al., 2008, 2009), and by introducing active linking and agent - based linking dynamics (Pachecho et al., 2006a, b; Traulsen et al., 2008). In particular Pachecho et al. (2006a, b) considered a population model in which the agents seek new connections at different rates, and allow the established connections to last for different amount of time. In the limit in which the dynamics of the network is much faster than the evolution of strategies the authors in particular show that the Prisoner's Dilemma game can be transformed to a coordination game - the transformation changes the rules of the game and explains the emergence of cooperation in the considered model. Pachecho et al. (2008) studied the systems with repeated interactions which last as long as the link between the players is present, and obtained analytical conditions for evolutionary stability under direct reciprocity. In comparison with the similar model on static graph (Ohtsuki and Nowak, 2007), the cooperation is facilitated by the active linking dynamics (in both cases the cooperation is promoted if the links last long enough, and the incentive to create new links is not too high).

We propose an approach, based on another idea of changing the connectivity structure in the system. We assume that the players dynamically change the connection weights, using update rules which reflect the tendency to increase more profitable connections, and to weaken the disadyantageous ones in a continuous way. The weights can be symmetric or asymmetric. In the first case the value of the connection is the same for both players, whereas in the second this value can be different for each of them.

We propose two types od models: finite population on a network, and continuous population in the mean field approximation. In the model of agents on network the agents are located on a random graph. Each agent is connected with some other agents (neighbors). Each connection has a weight, which changes according to preferences of the agent. The preferences of a player are measured by the difference between its payoff from the consid- 
ered connection and an averaged payoff. Evolution of the model occurs by a birth - death mechanism. In the mean field model the weights of the connections are time - dependent functions which evolve according to the rules of evolutionary game theory. Solutions of the resulting systems of differential equations are discussed. The asymptotic equilibrium states are investigated. For both models we study temporal evolution of the strategies and the weights distribution for two types of 2-person games describing the standard social dilemmas: the Prisoner's Dilemma game and the Snow Drift game.

\section{MODEL ON GRAPH}

The population consists of $\mathrm{N}$ agents, identified with the nodes of a random connected graph with a degree of $\mathrm{k}$. The edges are described by dynamically changing connection weights: we denote $\omega_{i j}(t)$ - the connection weight between the node $i$ and $j$ at time $t$. The connection weights can be symmetric: $\omega_{i j}(t) \equiv \omega_{j i}(t)$, or asymmetric, when $\omega_{i j}(t)$ and $\omega_{j i}(t)$ are in general different. The agents interact pairwise, playing a two - person symmetric game with the payoff matrix

$$
\begin{array}{l|ll} 
& \mathrm{C} & \mathrm{D} \\
\hline \mathrm{C} & \mathrm{a} & \mathrm{b} \\
\mathrm{D} & \mathrm{c} & \mathrm{d}
\end{array}
$$

(abbreviated in the description of figures by $[a, b, c, d]$ ) with their neighbors, using the strategy $\mathrm{C}$ or $\mathrm{D}$, and receive payoffs which are products of the payoffs from the above payoff matrix, and the relevant connection weights. We shall refer to such products as to the effective payoffs. In our paper $\mathrm{C}$ stands for cooperation and $\mathrm{D}$ for defection in the considered below social dilemma games, although in general the proposed scheme is valid for any 2-person game, and can be generalized for other types of the games.

Initially the agent's strategies are allocated randomly. The initial connection weights are allocated in such a way that the assumed order of the graph is obtained, and typically are the same for all connected players.

The evolution of the network takes place in discrete time steps. At each time step first the weights of all the connections are updated, then the strategy of one of the agents and its weights are updated.

The updating rules will reflect the fact that each player tends to increase the intensity of interactions (the connection weights in our model) with those opponents with whom the 
results of the interactions, i.e. the effective payoffs are higher than the average payoff from all the interactions of the player. The increase is proportional to the difference between both types of payoffs. The rules of the weights updating in the symmetric and asymmetric model are respectively:

$$
\begin{gathered}
\omega_{i j}(t+1)=\omega_{i j}(t)+\omega_{i j}(t)\left(1-\omega_{i j}(t)\right)\left(\Delta_{i j}+\Delta_{j i}\right) / 2 M \\
\omega_{i j}(t+1)=\omega_{i j}(t)+\omega_{i j}(t)\left(1-\omega_{i j}(t)\right) \Delta_{i j} / M
\end{gathered}
$$

where $\Delta_{i j}$ is the difference between the effective payoff of the agent in the node $i$ from the interaction with that in $j$ (given by the product of $\omega_{i j}$ and the relevant entry of the payoff matrix introduced above), and the mean payoff of the agent in $i$ over the neighborhood of $i$. Formally $\Delta_{i j}$ is defined as follows. Let $d_{i j}$ denotes the payoff of the $i$ player from the interaction with the $j$ player, calculated from the relevant entry of the initial payoff matrix (1). Let $N_{i}$ denotes the number of the $i$-th neighbors, i.e. the nodes connected to $i$. Then $\Delta_{i j}(t)=\omega_{i j}(t) d_{i j}-\frac{1}{N_{i}} \sum_{l=1}^{l=N_{i}} \omega_{i l}(t) d_{i l}$. $M$ denotes the maximum of the payoff matrix.

The product $\omega_{i j}\left(1-\omega_{i j}\right)$ in $(1)$, (2) reduces the speed of the evolution of the relevant weight when it approaches its extremal values (here normalized to zero and unity). In other words, the ties which are close to their extremal values are harder to change.

Strategy updating for both the symmetric and antisymmetric weights models is based on the BD (birth - death) method (Ohtsuki and Nowak, 2006). We consider two types of updates, which we call no inheritance and inheritance updates. In the former we draw a player (parent), with probability proportional to its total payoff. Then we draw randomly one of its neighbors (descendant), and allocate to it the parent's strategy. The weights of the descendant with its neighbors are set to their initial values. In the latter the strategy is inherited as above; the connection weights between the neighbors and the descendant are calculated from the neighbor's and the parent mean values. Each player can have two, in general different, mean values of the connection weights: one calculated from the interactions with partners who play $\mathrm{C}$ and one with those who play $\mathrm{D}$. In the inheritance symmetric model the weights neighbors-descendant are equal to the relevant mean values of the parent. In the inheritance asymmetric model the weights descendant-neighbors are equal to the mean values of the parent's weights, the weights neighbors-descendant are for each neighbor equal to the relevant mean values of the neighbor's weights. 
The proposed model belongs to the class of the coevolutionary models with two characteristic time scales: the first one characterizes the frequency of strategy updates, the second one describes the frequency of changes of the weights. In many biological applications it has been assumed that the time scale of the interactions between the individuals is much shorter that the time scale of the selection processes. The dependence of the results, in particular of the maintenance of coordination in the long run, on the scaling of these two processes, was studied by various authors. In particular Santos et al. (2006) show that for a given average connectivity of the population, there is a critical value of the ratio between the time scale associated with the evolution of strategies and of the network connectivity structure, above which the cooperation is maintained in the system. The problem of time scales for different processes in the coevolutionary models was also considered e.g. by Pachecho et al. (2006a, b), and Roca et al. (2006). Our analysis is restricted to the situations in which the characteristic time scale of the strategy updating is much bigger then that of the connection updating.

\section{MEAN FIELD MODEL}

We assume the evolutionary scenario in which each agent interacts with the other agents through a random pairwise matching, playing at each instant of time a 2- person symmetric game with the payoff matrix $\left[\begin{array}{ll}a & b \\ c & d\end{array}\right]$.

Let $\mu=\mu(t)$ denotes frequency of agents playing $C$ in the whole population. We introduce $\omega_{F S} \equiv \omega_{F S}(t)$ - the weight of the connection between the agent playing strategy $F$ and that playing $S$, and denote: $U_{F S}$ - the effective payoff of the $F$ - agent from the interaction with the $S$ - agent, $F, S \in\{C, D\}$ :

$$
U_{C C}=a \omega_{C C}, U_{C D}=b \omega_{C D}, U_{D C}=c \omega_{D C}, U_{D D}=d \omega_{D D}
$$

$U_{C}, U_{D}$ - the effective payoffs of respectively $C$ and $D$ - agents:

$$
U_{C}=\mu U_{C C}+(1-\mu) U_{C D}, \quad U_{D}=\mu U_{D C}+(1-\mu) U_{D D}
$$

and $U$ - the effective payoff of an agent in the population:

$$
U=\mu U_{C}+(1-\mu) U_{D}
$$

In general the effective payoff matrix, with the entries defined by (3), evolves in time, and its time asymptotic form is different from the initial one, as will be discussed below. 
The change of the frequency $\mu$ of the strategy $C$ in the population is assumed to be governed by the evolution equation

$$
\dot{\mu}=\mu\left(U_{C}-U\right)
$$

In the symmetric weights model we assume that $\omega_{C D}=\omega_{D C}$, and that the weights $\omega_{i j}, i, j \in\{C, D\}$ evolve according to the equations

$$
\dot{\omega}_{i j}=f\left(\omega_{i j}\right) \frac{\left(U_{i j}-U_{i}\right)+\left(U_{j i}-U_{j}\right)}{2}
$$

where $f(x)=x(1-x), x \in(0,1)$.

With notation $x=\omega_{C C}, y=\omega_{C D}=\omega_{D C}, z=\omega_{D D}$ we rewrite $(6),(7)$ as

$$
\begin{aligned}
\dot{x} & =x(1-x)(1-\mu)(a x-b y) \\
\dot{y} & =y(1-y)[\mu(b y-a x)+(1-\mu)(c y-d z)] / 2 \\
\dot{z} & =z(1-z) \mu(d z-c y) \\
\dot{\mu} & =\mu(1-\mu)\{\mu(a x-c y)+(1-\mu)(b y-d z)\}
\end{aligned}
$$

with a variety of the stationary points, cf. section $\mathrm{V}$.

In the asymmetric weights model in general $\omega_{C D} \neq \omega_{D C}$. We assume that the evolution of $\omega_{i j}$ is governed by

$$
\dot{\omega}_{i j}=f\left(\omega_{i j}\right)\left(U_{i j}-U_{i}\right), i \neq j, i, j \in\{C, D\}
$$

whereas $\mu$ evolves according to (6). The resulting system of equations is five dimensional.

\section{RESULTS: MODEL ON GRAPH}

We performed extensive simulations for different system sizes, graph degrees and payoff matrices. Below report results for the system size $N=500$, the graph degree $k=10$ or $k=N$ for the Prisoner's Dilemma PD game $\left[\begin{array}{ll}3 & 1 \\ 5 & 2\end{array}\right]$, and the Snow Drift SD game $\left[\begin{array}{ll}4 & 3 \\ 5 & 1\end{array}\right]$. More detailed study of other games, comparison of different models and results for other ratios of the characteristic time scales will be presented elsewhere.

In order to assure possibility of the temporal evolution of all the connections (i.e. to assure that the players can change the values of all the connection weights) it is assumed that all the entries of the initial payoff matrices are positive. Otherwise, an entry zero in the payoff matrix would result in zero value of the relevant effective payoff, independently of 
the current value of the weight associated with the entry. Thus, even thought the weight would evolve in time, the effective payoff associated to this weight would not change.

We denote $\mu_{0}$ - the initial, and $\mu_{\infty}$ - the time asymptotic value of $\mu$. All the initial weights are set equal to $\omega_{0}$. Results are averaged over 50 runs.

For the symmetric weights we obtain for the PD game the coexistence or dominance of the cooperators: for the no inheritance update rule and $k=10$ there are two asymptotic frequencies of C-players: $\mu_{\infty} \approx \frac{2}{5}$ and $\mu_{\infty}=1$, cf. Fig. 1a. Increasing degree of the graph

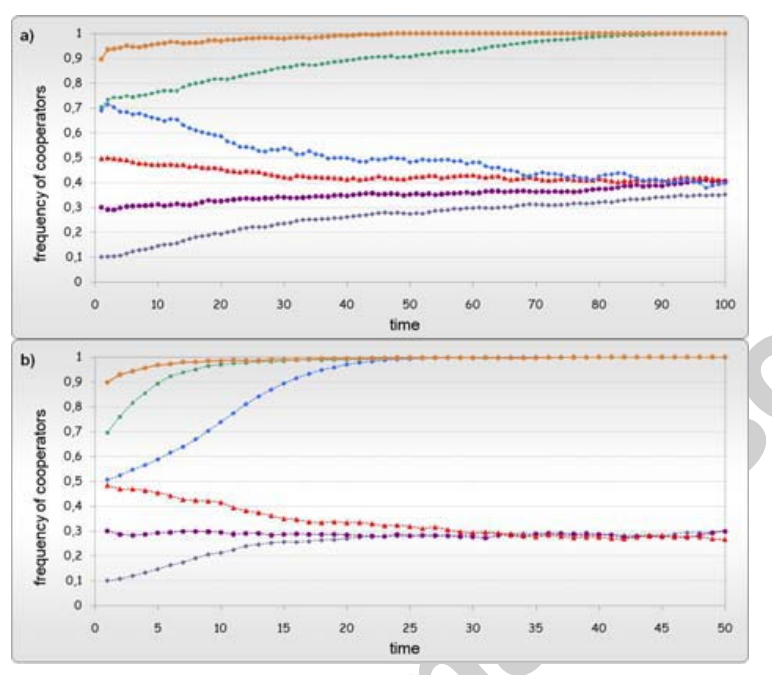

FIG. 1: $N=500, k=10, \omega_{0}=\frac{1}{2}$, symmetric weights; a: no inheritance, b: inheritance update, PD game

promotes C-players. In particular, for $k=N$ we obtain $\mu_{\infty}=1$ (not shown). For the inheritance update rule $\mu_{\infty} \approx \frac{1}{3}$ and $\mu_{\infty}=1$, cf. Fig. $1 \mathrm{~b}$.

For the PD game with the asymmetric weights we obtain $\mu_{\infty}=0$, i.e. the defectors always win for both update rules (not shown). $\omega_{D C}$ grows faster then $\omega_{C C}$, therefore in short time the defectors receive the payoffs high enough to take over the whole population.

For the SD game we present the diagram for the symmetric weights and the inheritance update. We obtain $\mu_{\infty} \approx 0.35$ and $\mu_{\infty}=1$, cf. Fig. 2. For the asymmetric weights with no inheritance update $\mu_{\infty} \approx 0.3$, whereas with the inheritance update $\mu_{\infty} \approx 0.7$, i.e. higher than the fraction of cooperators in the mixed Nash equilibrium $\mu_{\infty}=\frac{2}{3}$ for this game. 


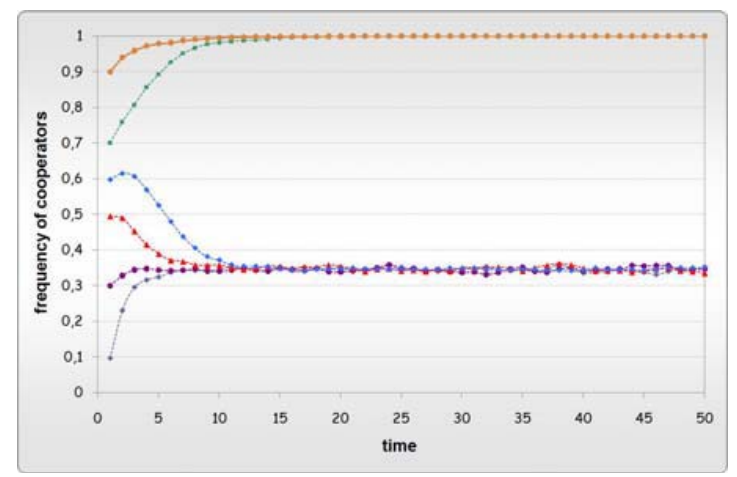

FIG. $2: N=500, k=10, \omega_{0}=\frac{1}{2}$, symmetric weights, inheritance update, SD game

\section{RESULTS: MEAN FIELD MODEL}

We calculated analytically the stationary points of the systems (8)-(11), and (6), (8),(10), (12). For example, for the PD matrix $\left[\begin{array}{ll}3 & 1 \\ 5 & 2\end{array}\right]$ used in the simulations, for the symmetric model the stationary point $\left(\omega_{C C}, \omega_{C D}, \omega_{D D}, \mu\right)=\left(1,1,0, \frac{1}{3}\right)$ is stable, and corresponds to the mixed equilibrium (i.e. with the fraction of cooperators $\left.\mu_{\infty} \in(0,1)\right)$. There are also two other stable equilibria, for which $\mu_{\infty}=0$, and $\mu_{\infty}=1$. For the asymmetric model the unique stable stationary point, corresponding to the mixed equilibrium of the considered PD game is $\left(\omega_{C C}, \omega_{C D}, \omega_{D C}, \omega_{D D}, \mu_{\infty}\right)=\left(0,1,1,0, \frac{1}{6}\right)$. The two other stable equilibria correspond to $\mu_{\infty}=0, \mu_{\infty}=1$. For the $\mathrm{SD}$ game $\left[\begin{array}{ll}3 & 2 \\ 5 & 1\end{array}\right]$ with symmetric weights, the locally stable equilibrium points have the $\mu_{\infty}$ coordinate $\frac{1}{2}, \frac{2}{7}$ or 1 . More systematic study of the solutions of the proposed model for different games, and analytical properties of the relevant equilibrium states will be presented elswhere.

We solved numerically the above systems of equations for different payoff matrices. In Fig. 3 we present an example of time evolution of $\omega_{i j}(t)$ and $\mu(t)$ for symmetric weights for the PD game $\left[\begin{array}{ll}3 & 1 \\ 5 & 2\end{array}\right]$ with $\omega_{0}=0.75, \mu_{0}=0.9$. The change of the fraction of cooperators is correlated with the evolution of the connection weights $\omega_{C D}(t), \omega_{D D}(t)$ and $\omega_{C C}(t)$.

Let us now define the effective payoff matrix at time $t$, in agreement with the notation introduced in (3), as $P_{t}=\left[\begin{array}{cc}a \omega_{C C}(t) & b \omega_{C D}(t) \\ c \omega_{D C}(t) & d \omega_{D D}(t)\end{array}\right]$. In stationary states the weights do not change, and the agents play a 2-person game defined by the effective payoff matrix $P_{\infty}=$ $\lim _{t \rightarrow \infty} P_{t}$, which in general is different from the initial one.

In Figs. 4, 5 we present the transition diagrams, which show how the type of the asymptotic effective payoff matrix $P_{\infty}$ depends on the initial data $\left(\omega_{0}, \mu_{0}\right)$ and on the type of 
the initial payoff matrix. The initial matrices belong to the one - parameter family of the matrices $\left[\begin{array}{ll}3 & 1+\alpha \\ 5 & 2-\alpha\end{array}\right], \alpha \in[0,2)$; in patricular $\alpha \in\left[0, \frac{1}{2}\right)$ correspond the PD games, $\alpha=\frac{1}{2}$ defines the 'intermediate' Weak PD game, $\alpha \in\left(\frac{1}{2}, 2\right)$ describe the SD games.

In Figs. 4, 5 each point $\left(\omega_{0}, \mu_{0}\right)$ of the squares $[0,1] \mathrm{x}[0,1]$ belongs to one of the domains defined below. Different domains characterize the type of the final effective payoff matrix obtained from the initial PD or SD game with the initial data $\left(\omega_{0}, \mu_{0}\right)$. For example, for the initial matrix $\left[\begin{array}{ll}3 & 2 \\ 5 & 1\end{array}\right], P_{\infty}$ is of the SD type: $\left[\begin{array}{cc}b-c / 2 & b-c \\ b & 0\end{array}\right]$ with $b=5$ and $c=4$. It represents the social dilemma in which cooperation implies a benefit $\mathrm{b}$ to the cooperator and to the opponent, and incurs a cost $c$ if the opponent defects, or $\frac{c}{2}$ if she cooperates, with the mixed strategy Nash equilibrium $\mu=\frac{2(b-c)}{2 b-c}$.

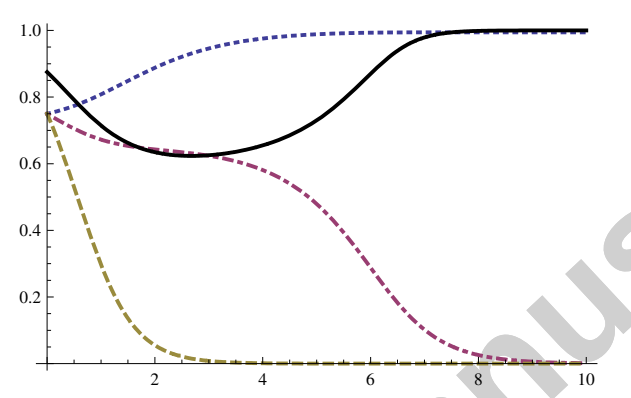

FIG. 3: $\mu(t)$ and $\omega_{F S}(t)$ for PD game $[3,1,5,2], \mu_{0}=0.9, \omega_{0}=0.75$. Lines: continuous: $\mu$, dotted: $\omega_{C C}$, dashed: $\omega_{D D}$, dotted-dashed: $\omega_{C D}$

The domain $\mathbf{C}$ describes the systems for which $P_{\infty}=\left[\begin{array}{cc}K & 0 \\ 0 & 2-\alpha\end{array}\right], K \in(0,3)$, and $\mu_{\infty}=1$ for all initial data (coordination game), PD - the systems for which $P_{\infty}=\left[\begin{array}{cc}3 & 1+\alpha \\ 5 & L\end{array}\right], L \in$ $(1+\alpha, 3)$, and $\mu_{\infty}=0$ for all initial data (prisoner's dilemma game), SD - the systems for which $P_{\infty}=\left[\begin{array}{cc}3 & 1+\alpha \\ 5 & 0\end{array}\right]$ (snow drift game), and AC - the systems for which $P_{\infty}=$ $\left[\begin{array}{cc}0 & 1+\alpha \\ 5 & 0\end{array}\right], \alpha \in(0,2)$ (anti-coordination game). The asymptotic frequency of cooperators $\mu_{\infty}$ in the domains $\mathbf{S D}$ and $\mathbf{A C}$ are equal to the relevant mixed Nash equilibrium values, see below.

For the initial PD matrix $\left[\begin{array}{ll}3 & 1 \\ 5 & 2\end{array}\right]$ (i.e. $\alpha=0$ ) there are three types of final payoff matrices, cf. Fig. $4 \mathrm{a}$, in which in the domain SD $\mu_{\infty}=\frac{1}{3}$. Increase of $\alpha$ (i.e. increase of the payoff of $\mathrm{C}$ against $\mathrm{D}$, accompanying by the simultaneous decrease of the payoff of $\mathrm{D}$ against D) results in shrinking of the $\mathrm{PD}$ domain and the emergence of the $\mathrm{AC}$ domain. This is illustrated, for $\alpha=\frac{1}{4}$, in Fig. 4b, in which in SD $\mu_{\infty}=\frac{5}{13}$, in $\mathrm{AC} \mu_{\infty}=\frac{1}{5}$. 


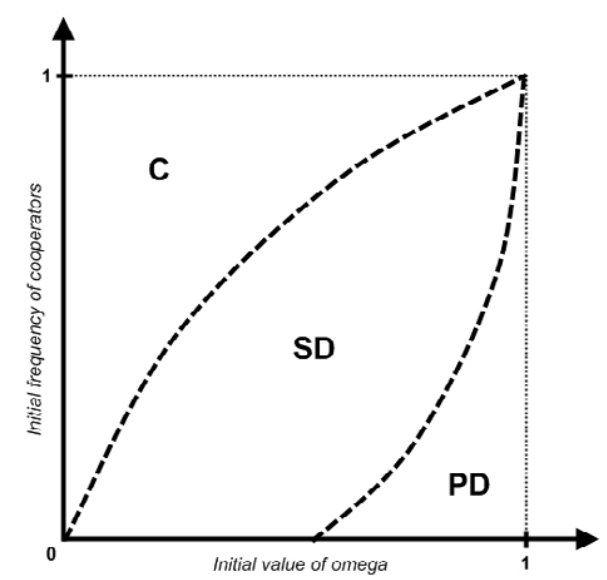

a)

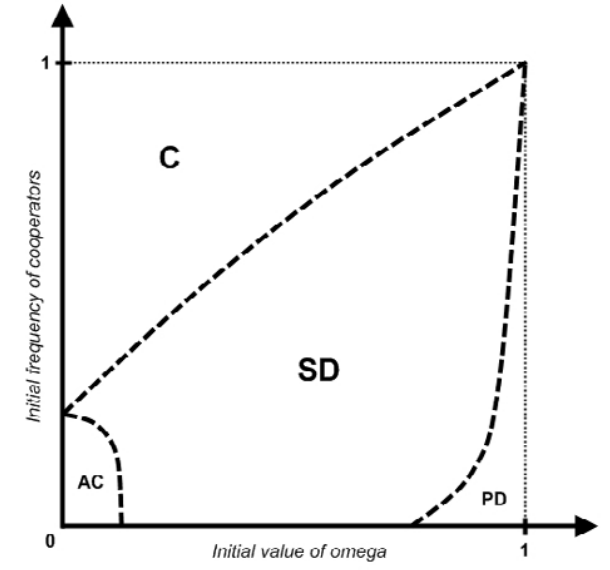

b)

FIG. 4: Transition diagrams for the mean field model, symmetric weights; a): PD initial matrix $[3,1,5,2] ;$ b): PD initial matrix $\left[3,1 \frac{1}{4}, 5,1 \frac{3}{4}\right]$

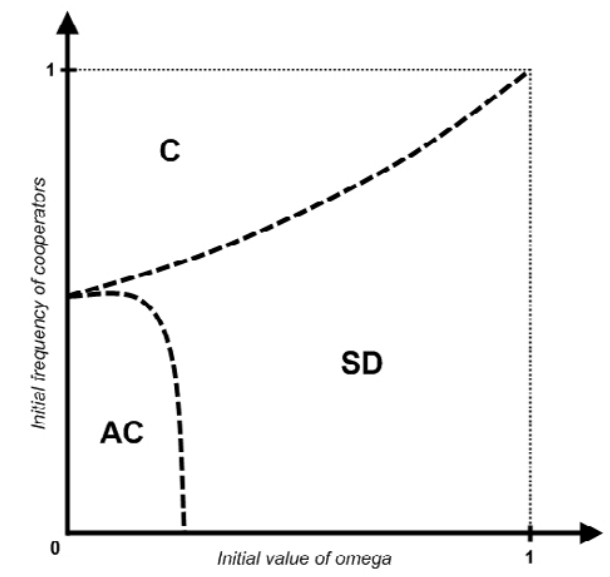

a)

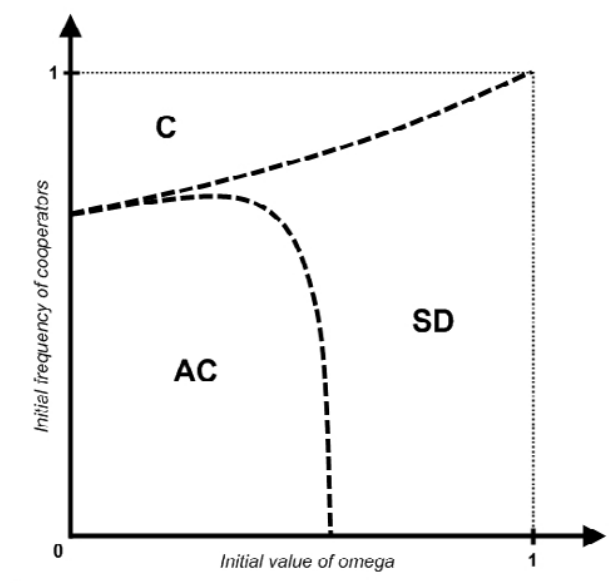

b)

FIG. 5: Transition diagrams for the mean field model, symmetric weights; a): Weak PD initial matrix $\left.\left[3,1 \frac{1}{2}, 5,1 \frac{1}{2}\right] ; \mathrm{b}\right)$ : SD initial matrix $[3,2,5,1]$

Further increase of $\alpha$ leads - for $\alpha=\frac{1}{2}$ - to the Weak PD game, cf. Fig. 5a, in which in $\mathbf{S D} \mu_{\infty}=\frac{3}{7}$, in $\mathbf{A C} \mu_{\infty}=\frac{3}{13}$. For $\alpha>\frac{1}{2}$ the initial game is SD. In Fig. $5 \mathrm{~b} \alpha=1$, and in SD $\mu_{\infty}=\frac{1}{2}$, in $\mathbf{A C} \mu_{\infty}=\frac{2}{7}$, with absence of the $\mathbf{P D}$ domain and enlargening of the AC domain. In the limiting case $\alpha=2$ corresponding to the 'weak' anticoordination 
game the final payoff matrix is always of the type $\mathrm{AC}$, i.e. all the square of initial $\left(\omega_{0}, \mu_{0}\right)$ is covered by one domain AC (not shown). Note also that, as expected, the increase of $\alpha$, i.e. the payoff of the cooperator from the interaction with the defector, implies the increase of the final percentage of the cooperators in the corresponding domains SD and AC.

For other initial PD games the topology of the transition diagrams is similar. The type, the number of the final domains and the points of intersection of the dotted lines with the axis depend on the numerical values of the payoffs, as discussed in the presented diagrams. We note that a similar change of the matrix types was found by Pachecho et al. (2006b), cf. also Traulsen et al. (2008). The authors proposed a mean field type model in which the evolution of the number of different types (CC, CD, DD) of the links was governed by a system of ordinary differential equations. In some well defined limits, under constraints for the initial state, the initial PD game was converted to a coordination game, and the $\mathrm{SD}$ game to a harmony game. While in the above papers the interactions advantageous for both interacting partners were assumed to last longer then when one player was exploited by the opponent in the symmetric situation, in our approach the advantageous weights are continuously strengthened, with the rate depending on their actual values.

For the asymmetric weights and the PD $\left[\begin{array}{ll}3 & 1 \\ 5 & 2\end{array}\right]$ we obtained $\mu_{\infty}=0$, and two forms of $P_{\infty}$ matrix: $P_{\infty}=\left[\begin{array}{ll}3 & \alpha \\ 5 & \beta\end{array}\right], 0<\alpha<\beta<2$ (PD games), or $P_{\infty}=\left[\begin{array}{ll}3 & 0 \\ 5 & 0\end{array}\right]$ (Weak PD game). For the SD initial matrix $\left[\begin{array}{ll}3 & 2 \\ 5 & 1\end{array}\right]$ we obtained $\mu_{\infty}=0$, and $P_{\infty}=\left[\begin{array}{ll}3 & 0 \\ 5 & 0\end{array}\right]$ (Weak PD), as the only type of the final payoff matrix (not shown). Thus, for both PD and SD the asymmetric weights lead to defection in the mean field model. For other initial PD and SD payoff matrices $\mu_{\infty}=0$, and $P_{\infty}$ have in general the same structure.

\section{DISCUSSION}

We introduced a new mechanism of the evolution of interactions between players, in which their connection weights are time dependent. We proposed two models in which the new mechanism is incorporated: the model of agents on graphs and the mean field model. In the model on graphs the speed of the changes of the player's connection weights is much faster than the speed of their strategy changes, and that makes the evolution of the weights the key factor in the coevolution of the whole system. In both models the dynamics of the strategy changes is governed by evolutionary rules of better fitness. The 
dynamical weights change the effective payoffs during the evolution, which may result in optimal coordination of the actions of the agents due to the interplay between the composition of the population and the strength of the effective links. In the case of the $\mathrm{PD}$ game the coexistence of both strategies in the long run is observed.

The proposed mechanism of the dynamics of the connections is quite general. The players know only their effective payoffs from the interactions, without knowing which game they participate at. They do not maximize their effective payoffs, but rather increase the weights of those connections which give them higher then average payoffs. The updating rules for the weights do not promote a priori any particular strategy. In the analogous models in which the connections between the agents are fixed the evolution leads to total defection for the PD game: in the graph model it follows from the graph structure and our strategy updating mechanism, whereas the mean field model is reduced to the classical replicator. More detailed study of other games, comparison of different models and results for other ratios of the characteristic time scales will be presented elsewhere. Further research topics include non symmetric matrices, evolution of different populations, games with more strategies and multi - player games.

Acknowledgements. We thank the anonimous reviewers for their useful comments. TP was supported by the Polish Government Grant no. N201 023 31/2069

\section{References}

Ashlock, D., Smucker, M.D., Stanley, E.A., Tesfatsion, L., 1996. Preferential partner selection in an evolutionary study of the Prisoner's Dilemma. BioSystems 37, 99-125

Ebel, H., Bornholdt, S., 2002. Coevolutionaty games on networks. Phys. Rev. E 66, 056118

Eguiluz V. M., Zimmermann M. G., Cela-Conde, C.J., San Miguel M., 2005. Cooperation and the emergence of role differentiation in the dynamics of social networks, Am. J. Soc. 110, 977-1008

Gross, T., Blasius, B., 2008. Adaptive coevolutionary networks: a review. J. R. Soc. Interface 5, 20, 259-271

Hauert, C., Szabo, G., 2003. Prisoner's dilemma and public good games in different geometries: compulsory versus voluntary interactions. Complexity 8, 31-38

Nowak, M.A., May, R.M., 1992. Evolutionary games and spatial chaos. Nature 359, 826-829

Ohtsuki H., Nowak M.A., 2006. The replicator equations on graphs. J. Theor. Biol. 243, 86-97

Ohtsuki, H., Nowak, M.A., 2007. Direct reciprocity on graphs. J. Theor. Biol. 247, 462-470

Pachecho, J.M, Traulsen, A., Nowak, M.A., 2006a. Active linking in evolutionary games. J. Theor. Biol. 243, 437-443

Pachecho, J.M, Traulsen, A., Nowak, M.A., 2006b. Coevolution of Strategy and Structure in Complex Networks with Dynamical Linking. Phys. Rev. Lett. 97, 258103

Pachecho, J.M, Traulsen, A., Ohtsuki, H., Nowak, M.A., 2008. Repeated games and direct reciprocity under active linking. J. Theor. Biol. 250, 723-731

Poncela, J., Gomes-Gardenes, Floria, L.M., Sanchez, A., Moreno, Y., 2007. PLoS One 3, e2449

Roca, C.P., Cuesta, J.A., Sanchez, A., 2006. Time Scales in Evolutionary Dynamics. Phys. Rev. Lett. 97,158701 
Santos, F.C., Pachecho, J.M., Lenaerts, T., 2006. Cooperation Prevails When Individuals Adjust Their Social Ties. PLoS Comput. Biol. 2, 1284

Szabo, G., Fath, G., 2007. Evolutionary games on graphs. Physics Reports 446 (4-6), 97-216

Traulsen, A., Santos, F.C., Pachecho, J.M., 2008. Evolutionary games in self-organizing populations. In "Adaptive Networks: Theory, Models and Applications", T. Gross \& H. Sayama Eds., New England Complex Systems Book Series on Complexity, Springer N.Y.

Van Segbroeck, S., Santos, F.C., Pachecho, J.M., Lenaerts, T., 2008. The evolution of prompt reaction to adverse ties. BMC Evolutionary Biology 8:287

Van Segbroeck, S., Santos, F.C., Pachecho, J.M., Lenaerts, T., 2009. Reacting differently to adverse ties promotes cooperation in social networks. Phys. Rev. Lett. 102, 058105

Zimmermann M.G., Eguiluz V.M., San Miguel M., 2004. Coevolution of dynamical states and interactions in dynamic networks. Phys. Rev. E 69, 065102(R)

Zimmermann M. G., Eguiluz V.M., 2005. Cooperation, social networks and the emergence of leadeship in a prisoner's dilemma with adaptive local interactions. Phys. Rev. E 72, 056118 